\title{
In vitro antimalarial activity of tigecycline against Plasmodium falciparum culture-adapted reference strains and clinical isolates from the Brazilian Amazon
}

\author{
Daniele Ribatski-Silva ${ }^{[1]}$, Carmen Lucia Bassi ${ }^{[2]}$, Thamires Oliveira Gasquez Martin ${ }^{[1]}$, \\ Eduardo Alves-Junior ${ }^{[1]}$, Luciano Teixeira Gomes ${ }^{[1],[3]}$ and Cor Jésus Fernandes Fontes ${ }^{[1],[3],[4]}$
}

[1]. Pós-Graduação em Ciências da Saúde, Faculdade de Medicina, Universidade Federal de Mato Grosso, Cuiabá, MT. [2]. Laboratório de Investigação, Faculdade de Medicina, Universidade Federal de Mato Grosso, Cuiabá, MT. [3]. Grupo de Estudos de Doenças Infecciosas e Tropicais de Mato Grosso, Universidade Federal de Mato Grosso, Cuiabá, MT. [4]. Faculdade de Medicina, Faculdade de Ciências Biomédicas de Cacoal, Cacoal, RO.

\begin{abstract}
Introduction: We evaluated the in vitro antimalarial activity of tigecycline as an alternative drug for the treatment of severe malaria. Methods: A chloroquine-sensitive Plasmodium falciparum reference strain, a chloroquine-resistant reference strain, and three clinical isolates were tested for in vitro susceptibility to tigecycline. A histidine-rich protein in vitro assay was used to evaluate antimalarial activity. Results: The geometric-mean 50\% effective concentration (EC50\%) of tigecycline was $535.5 \mathrm{nM}$ (confidence interval (CI): 344.3-726.8). No significant correlation was found between the EC50\% of tigecycline and that of any other tested antimalarial drug. Conclusions: Tigecycline may represent an alternative drug for the treatment of patients with severe malaria.
\end{abstract}

Keywords: Plasmodium falciparum. In vitro susceptibility. Tigecycline.

In combination with traditional antimalarial drugs, antibiotics with antimalarial activity, such as tetracyclines and clindamycin, are recognized options for the treatment of multidrug-resistant Plasmodium falciparum malaria ${ }^{1-3}$. In recent decades, doxycycline has been included in the World Health Organization Model List of Essential Medicines as an antibacterial agent for the prevention and treatment of malaria ${ }^{4}$. The development of additional antimicrobial drugs has led to the discovery of glycylcyclines, which are tetracycline derivatives containing a glycylamido substitution at position nine ${ }^{5}$. Tigecycline, the first marketed compound of the glycylcycline class, is a semisynthetic derivative of minocycline that is specifically designed to overcome two common mechanisms of tetracycline resistance, including resistance mediated by acquired efflux pumps and ribosomal protection ${ }^{6}$. Pharmacological functional assays have shown that tigecycline inhibits bacterial protein synthesis with a potency that is 3 - and 20-fold greater than that of minocycline and tetracycline, respectively ${ }^{5}$. This drug is currently registered only for intravenous use, and a twice-daily dosing regimen is relatively easy to administer and is generally well tolerated ${ }^{7}$. Clinical studies have shown that tigecycline possesses an expanded spectrum of in vitro and in vivo activity against Gram-positive, Gram-negative, atypical, anaerobic, and

\footnotetext{
Address to: Drª Daniele Ribatski-Silva. Pós-Graduação Ciências da Saúde/FM/ UFMT. Av. Fernando Correa da Costa 2367, Bairro Boa Esperança, 78060-900 Cuiabá, MT, Brasil.

Phone: 5565 3615-7253

e-mail: dribatski@hotmail.com

Received 2 October 2012

Accepted 9 February 2013
}

other difficult-to-treat pathogens ${ }^{8}$. However, there is insufficient information on the drug's clinical efficacy for malaria treatment, as only two previous studies have demonstrated in vitro antiP. falciparum activity for this drug ${ }^{9,10}$. Because tigecycline is an exclusively injectable antibiotic, its use in malaria treatment should be reserved for critically ill patients. Moreover, this drug should always be used in combination with fast-acting antimalarials, given its moderate-to-slow P. falciparum schizonticide effect ${ }^{10}$. To determine its suitability as an alternative drug for the treatment of severe malaria, we evaluated the in vitro antimalarial activity of tigecycline against chloroquine-sensitive and chloroquine-resistant reference strains of $P$. falciparum and clinical isolates from the Brazilian Amazon.

Plasmosium falciparum clinical isolates were obtained from patients with uncomplicated malaria presenting at the Malaria Research Unit of the Julio Muller University Hospital in Cuiabá, State of Mato Grosso, Brazil between March and December 2011. After informed consent was obtained, a blood sample was collected in a vacuum tube containing heparin and was properly processed within 30min of collection to adapt the parasites to a continuous culture system. The study protocol was approved by the Ethical Research Committee of the Julio Muller University Hospital, Cuiabá (Mato Grosso), Brazil. Patients were included if they demonstrated P. falciparum monoinfection; parasitemia above 250 parasites/ $\mu \mathrm{L}$ blood, as assessed by Giemsa-stained thick blood film; and no history of antimalarial drug treatment for at least 30 days.

Chloroquine-sensitive (3D7) and chloroquine-resistant (DW2) reference strains and the P. falciparum clinical isolates were kept in complete culture medium (RPMI 1640, 25mM HEPES, $2 \mathrm{mM}$ L-glutamine, $40 \mu \mathrm{g} / \mathrm{mL}$ gentamicin, $0.36 \mathrm{mM}$ hypoxanthine, $10 \mathrm{mM}$ glucose, and $10 \% \mathrm{v} / \mathrm{v}$ inactivated human 
TABLE 1 - In vitro susceptibilities of culture-adapted reference strains and clinical isolates of Plasmodium falciparum from the Brazilian Amazon to tigecycline.

Antimalarial activity*

$(\mathrm{nM})$

\begin{tabular}{lcc} 
Plasmodium falciparum strains/isolates & EC50\% & EC99\% \\
\hline DW2 & 568.9 & $13,095.3$ \\
3D7 & 332.0 & $19,459.5$ \\
HUJM1 & 674.6 & $22,426.2$ \\
HUJM3 & 678.6 & $18,675.3$ \\
HUJM2 & 423.4 & $18,605.2$ \\
Mean (CI95\%) & $535.5(344.3 ; 726.8)$ & $18,452.3(14,261.0 ; 22,643.6)$
\end{tabular}

*Determined using HRP2 in an immune-enzymatic assay; EC50\%: geometric-mean 50\% effective concentration; EC99\%: geometric-mean 99\% effective concentration. 95\% CI: 95\% confidence interval.

serum at $37^{\circ} \mathrm{C}, 5 \%$ oxygen, and $5 \%$ hematocrit. All parasitized cultures were synchronized by sorbitol treatment and seeded as ring stages before susceptibility assays were performed.

A histidine-rich protein 2 (HRP2) in vitro assay was performed to evaluate anti-P. falciparum activity. The HRP2 assays were performed according to standard procedures. Briefly, the culture-adapted $P$. falciparum isolates and the reference strains were cultured in the presence of 3-fold serial dilutions of the antimalarial drugs tigecycline $(332-21,250 \mathrm{nM})$, chloroquine (25-1,600nM), quinine (50-3,200nM), mefloquine (2.5-160nM), lumefantrine $(1.25-80 \mathrm{nM})$, artemisinin $(0.25-16 \mathrm{nM})$, and artesunate $(0.25-16 \mathrm{nM})$. After $72 \mathrm{~h}$ of culture, the plates were frozen and stored at $-20^{\circ} \mathrm{C}$. Chloroquine was dissolved in doubledistilled water; quinine, mefloquine, artemisinin, and artesunate were dissolved in methanol; lumefantrine was dissolved in ethanol-linoleic acid-Tween 80 mixture (v/v/v 1:1:1); and tigecycline was obtained from the commercial product Tygacil $\AA$ (Wyeth, MO, USA) and dissolved in phosphate-buffered saline. The plates were then thawed, and parasite growth inhibition was quantified using a highly sensitive HRP2 ELISA. The optical density was measured at 450nm using a standard Enzyme linked immunosorbent assay (ELISA) plate reader (iMarkTM, Bio-Rad, USA). The optical density readings were used to estimate the inhibitory concentrations by nonlinear regression analysis ${ }^{11}$.

Of 10 patients, only 3 were successfully tested. Overall, the geometric-mean $50 \%$ effective concentration (EC50\%) of tigecycline for the 3 culture-adapted isolates and the 2 reference strains was $535.5 \mathrm{nM}$ (95\% confidence interval (CI): $344.3-$ 726.8). The EC50\% and 99\% effective concentration (EC99\%) values for all tested drugs are listed in Table 1. The individual effective concentrations were calculated for all tested drugs in parallel and were correlated using nonparametric correlation analysis to determine potential cross-sensitivity and/or crossresistance patterns between the drugs at their EC50\% levels ${ }^{12}$. The EC50\% of tigecycline exhibited no significant correlation with any other tested antimalarial, suggesting different modes of action and the absence of cross-resistance/sensitivity among the drugs ${ }^{1}$. However, the high value of Spearman's correlation coefficient $(\mathrm{r}=0.831 ; \mathrm{p}=0.080)$ between tigecycline and chloroquine was of concern, as this coefficient may indicate the potential for cross-resistance between these drugs (Table 2).

Our findings demonstrate that tigecycline possesses in vitro antimalarial activity against chloroquine-sensitive and chloroquine-resistant culture-adapted isolates of $P$. falciparum, which is consistent with the findings of previous studies ${ }^{9,10}$. In general, patients with severe and complicated malaria are treated with artemether or artesunate by parenteral administration. To potentiate the schizonticide effect of these artemisinin derivatives, the co-administration of an injectable antibiotic, such as clindamycin ${ }^{13,14}$, is also recommended. As an exclusively parenterally administered antibiotic, tigecycline may represent an alternative drug for treating patients with severe and complicated $P$. falciparum malaria. However, in vivo assays and randomized clinical trials are needed to establish the true efficacy and clinical applicability of tigecycline.

TABLE 2 - Correlation between the EC50\% of tigecycline and standard antimalarial drugs against culture-adapted reference strains and clinical isolates of Plasmodium falciparum from the Brazilian Amazon.

\begin{tabular}{lcc}
\hline & \multicolumn{2}{c}{ Tigecycline } \\
& \multicolumn{2}{c}{$(\mathrm{nM})$} \\
\cline { 2 - 3 } Drugs & $\mathrm{r}(\mathrm{p}$ value $)$ & $\mathrm{r}(\mathrm{p}$ value $)$ \\
\hline Chloroquine & $0.831(0.080)$ & $-0.776(0.122)$ \\
Mefloquine & $0.035(0.954)$ & $-0.977(0.004)$ \\
Quinine & $0.567(0.318)$ & $-0.338(0.577)$ \\
Lumefantrine & $0.354(0.558)$ & $-0.980(0.003)$ \\
Artemisinin & $0.163(0.792)$ & $-0.527(0.361)$ \\
Artesunate & $-0.212(0.731)$ & $-0.422(0.479)$ \\
\hline
\end{tabular}

r: Spearman's correlation coefficient; EC50\%: geometric-mean 50\% effective concentration; EC99\%: geometric-mean 99\% effective concentration. 


\section{CONFLICT OF INTEREST}

The authors declare that there is no conflict of interest.

\section{FINANCIAL SUPPORT}

This study received support from the Brazilian National Scientific and Technological Research Council (CNPq); Programa de Apoio a Núcleos de Excelência (PRONEX)-Rede Malaria, Mato Grosso; the Pan-American Health Organization and Brazilian Ministry of Health; and the Amazon Network for the Surveillance of Antimalarial Drug Resistance (RAVREDA).

\section{REFERENCES}

1. Noedl H, Krudsood S, Leowattana W, Tangpukdee N, Thanachartwet $\mathrm{W}$, Looareesuwan S, et al. In vitro antimalarial activity of azithromycin, artesunate, and quinine in combination and correlation with clinical outcome. Antimicrob Agents Chemother 2007; 51:651-656.

2. Ramharter M, Noedl H, Winkler H, Graninger W, Wernsdorfer WH, Kremsner PG, et al. In vitro activity and interaction of clindamycin combined with dihydroartemisinin against Plasmodium falciparum. Antimicrob Agents Chemother 2003; 47:3494-3499.

3. Sponer U, Prajakwong S, Wiedermann G, Kollaritsch H, Wernsdorfer G, Wernsdorfer WH. Pharmacodynamic interaction of doxycycline and artemisinin in Plasmodium falciparum. Antimicrob Agents Chemother 2002; 46:262-264.

4. World Health Organization (WHO). Model List of Essential Medicines [Internet]. WHO; 2011 [Cited 2012 August 19]. Availble from: http://whqlibdoc.who.int/hq/2011/a95053_eng.pdf/.
5. Olson MW, Ruzin A, Feyfant E, Rush TS, O’Connell J, Bradford PA. Functional, biophysical, and structural bases for antibacterial activity of tigecycline. Antimicrob Agents Chemother 2006; 50:2156-2166.

6. Hawkey P, Finch R. Tigecycline: in vitro performance as a predictor of clinical efficacy. Clin Microbiol Infect 2007; 13:354-362.

7. Muralidharan G, Micalizzi M, Speth J, Raible D, Troy S. Pharmacokinetics of tigecycline after single and multiple doses in healthy subjects. Antimicrob Agents Chemother 2005; 49:220-229.

8. Wilke MH. Multiresistant bacteria and current therapy - the economical side of the story. Eur J Med Res 2010; 15:571-576.

9. Starzengruber P, Thriemer K, Haque R, Khan WA, Fuehrer HP, Siedl A, et al. Antimalarial Activity of Tigecycline, a Novel Glycylcycline Antibiotic. Antimicrob Agents Chemother 2009; 53:4040-4042.

10. Held J, Zanger P, Issifou S, Kremsner PG, Mordmüller B. In vitro activity of tigecycline in Plasmodium falciparum culture-adapted strains and clinical isolates from Gabon. Int J Antimicrob Agents 2010; 35:587-589.

11. Noedl H, Bronnert J, Yingyuen K, Attlmayr B, Kollaritsch H, Fukuda M. Simple histidine-rich protein 2 double-site sandwich enzyme-linked immunosorbent assay for use in malaria drug sensitivity testing. Antimicrob Agents Chemother 2005; 49:3575-3577.

12. Ringwald P, Bickii J, Basco LK. In vitro activity of Lumefantrine (Benflumetol) against clinical isolates of Plasmodium falciparum in Yaoundé, Cameroon. Antimicrob Agents Chemother 1998; 42: 2347-2351.

13. World Health Organization (WHO). Guidelines for the treatment of malaria [Internet]. Geneva: WHO Library Cataloguing 2010; [Cited 2012 September 19]. Available from: http://www.who.int/malaria/publications/ atoz/9789241547925/en/index.html/.

14. Ministério da Saúde. Guia prático de tratamento da malária no Brasil. Brasília: Ministério da Saúde; 2010. 